This is an author-posted version of the below publication. For more information see https://www.spiedigitallibrary.org/article-sharing-policies

Mats U. Persson, Lin Fu, Peter M. Edic, Bruno De Man, "A new series expansion method and its application to photon-counting CT reconstruction", Proc. SPIE 11312, Medical Imaging 2020: Physics of Medical Imaging; 113121H (16 March 2020) https://doi.org/10.1117/12.2549623

Copyright 2020 Society of Photo-Optical Instrumentation Engineers (SPIE). One print or electronic copy may be made for personal use only. Systematic reproduction and distribution, duplication of any material in this publication for a fee or for commercial purposes, and modification of the contents of the publication are prohibited. 


\section{A new series expansion method and its application to photon-counting CT reconstruction}

Persson, Mats, Fu, Lin, Edic, Peter, De Man, Bruno

Mats U. Persson, Lin Fu, Peter M. Edic, Bruno De Man, "A new series expansion method and its application to photon-counting CT reconstruction," Proc. SPIE 11312, Medical Imaging 2020: Physics of Medical Imaging, 113121H (16 March 2020); doi: 10.1117/12.2549623 


\title{
A new series expansion method and its application to photon-counting CT reconstruction
}

\author{
Mats U. Persson ${ }^{1}$, Lin $\mathrm{Fu}^{2}$, Peter M. Edic ${ }^{2}$, and Bruno De $\mathrm{Man}^{2}$ \\ ${ }^{1}$ Department of Physics, KTH Royal Institute of Technology, SE-10691, Stockholm, Sweden \\ ${ }^{2}$ GE Research, Niskayuna, NY 12309, USA
}

\begin{abstract}
The introduction of photon-counting detectors in x-ray computed tomography raises the question of how reconstruction algorithms should be adapted to photon-counting measurement data. The transition from energyintegrating to photon-counting detectors introduces new effects into the data model, such as pure Poisson statistics and increased cross talk between detector pixels, (e.g. due to charge sharing), but it is still not known in detail how these effects can be treated accurately by the reconstruction algorithm. In this work, we propose a new reconstruction method based on penalized-likelihood reconstruction that incorporates these effects. By starting from a simple, easily-solved reconstruction problem and adding correction terms for the additional physical effects, we obtain a series expansion for the solution to the image reconstruction problem. This approach serves the twofold purpose of (1) yielding a new, potentially faster method of incorporating complex detector models in the reconstruction process and (2) providing insight into the impact of the non-ideal physical effects on the reconstructed image. We investigate the potential for reconstructing images from simulated photon-counting energy-resolving CT data with the new algorithm by including correction terms representing pure Poisson statistics and interpixel cross talk; and we investigate the impact of these physical effects on the reconstructed images. Results indicate that using two correction terms gives good agreement with the converged solution, suggesting that the new method is feasible in practice. This new approach to image reconstruction can help in developing improved reconstruction algorithms for photon-counting CT.
\end{abstract}

Keywords: Photon counting, Image reconstruction, Iterative reconstruction, series expansion, cross-talk correction

\section{INTRODUCTION}

Photon-counting computed tomography is expected to be the next major advancement in computed tomography (CT) technology, showing potential for improved contrast-to-noise ratio, higher spatial resolution, improved material selective imaging, and more quantitatively-reliable image measurements. ${ }^{1,2}$ However, the full realization of these objectives will require development of novel reconstruction algorithms that can make optimal use of measured photon-counting data. This strategy involves modeling Poisson noise and intradetector cross talk accurately.

In order to realize the full potential of photon-counting $\mathrm{CT}$ to generate quantitatively reliable images, these effects have to be taken into account in the image reconstruction. In the framework of model-based iterative reconstruction (MBIR), this can be achieved by including an accurate model of each of the non-ideal physical effects in the forward model of the reconstruction process. However, this increases the computational complexity of the reconstruction algorithm.

In this work we propose a new method of reconstructing CT images from projection data. By first solving a simpler reconstruction problem and the adding small correction terms to this original problem representing physical effects, we obtain the solution to the original reconstruction problem as a Taylor series expansion. Although Taylor series approximations have been applied to the Poisson likelihood expression before, ${ }^{3}$ the proposed method is different in the sense that it represents the solution of the optimization problem using Taylor expansion rather than just the objective function. The benefits of the new method are twofold.

M. U. Persson: E-mail: mats.persson@mi.physics.kth.se

Medical Imaging 2020: Physics of Medical Imaging, edited by Guang-Hong Chen, Hilde Bosmans, Proc. of SPIE Vol. 11312, 113121H · @ 2020 SPIE · CCC code: 1605-7422/20/\$21 · doi: 10.1117/12.2549623 
First, it has the potential to yield a fast reconstruction algorithm because it allows complicated physical effects to be incorporated in the image reconstruction process as corrections instead of being included in the forward model of every iteration, which can reduce computational cost. Second, calculating correction factors attributable to a particular physical effect makes it possible to understand the impact of these effects on the properties of the final image. Such insight can be a valuable tool to guide the development of both reconstruction algorithms and detector configurations for photon-counting CT. We study the effects of replacing a Gaussian noise model with a pure Poisson model, using a spectral forward model and with interpixel cross talk taken into account, in a simulation model of a photon-counting detector.

\section{METHOD}

Let $\Phi^{1}(\boldsymbol{a})$ be an objective function of a set of basis material images $\boldsymbol{a}$. Here $\boldsymbol{a}=\left(a_{l j}\right)$ is indexed by two indices $l$ (basis material) and $j$ (reconstructed pixel index), such that the attenuation coefficient in pixel $j$ is $\mu(E)=\sum_{l} a_{l j} f_{l}(E)$ with $f_{l}(E)$ being the linear attenuation coefficient of basis material $l$ as a function of energy $E$. Although it is parameterized by two indices for notational convenience, we will treat $\boldsymbol{a}$ as a vector of $L J$ elements where $L$ is the number of basis functions and $J$ is the number of reconstructed image pixels. Instead of minimizing $\Phi^{1}(\boldsymbol{a})$, which may be computationally challenging, we will assume that there is an approximate objective function $\Phi^{0}(\boldsymbol{a})$ that can be solved easily, and obtain the minimum of $\Phi^{1}(\boldsymbol{a})$ by adding correction terms to the minimum of $\Phi^{1}(\boldsymbol{a})$.

The $\boldsymbol{a}$ that minimizes the objective function

$$
\Phi(\boldsymbol{a}, \epsilon)=\Phi^{0}(\boldsymbol{a})+\epsilon\left(\Phi^{1}(\boldsymbol{a})-\Phi^{0}(\boldsymbol{a})\right)
$$

defines a function $\hat{\boldsymbol{a}}(\epsilon)=\underset{\boldsymbol{a}}{\operatorname{argmin}} \Phi(\boldsymbol{a}, \epsilon)$. The idea is now to calculate $\hat{\boldsymbol{a}}(1)$ by Taylor series expansion of $\hat{\boldsymbol{a}}(\boldsymbol{\epsilon})$ as $\hat{\boldsymbol{a}}(\boldsymbol{\epsilon})=\sum_{n=0}^{\infty} \hat{\boldsymbol{a}}^{(n)}(0) \epsilon^{n} / n$ ! and setting $\epsilon=1$. The derivatives $\hat{\boldsymbol{a}}^{(n)}$ can be calculated implicitly by differentiating $\Phi(\hat{\boldsymbol{a}}, \epsilon)$, making use of the fact that $\frac{\partial}{\partial a_{l_{1}, j_{1}}} \Phi(\hat{\boldsymbol{a}}, \epsilon)=0$ :

$$
\begin{gathered}
\sum_{l_{2}, j_{2}} \frac{\partial^{2}}{\partial a_{l_{1}, j_{1}} \partial a_{l_{2}, j_{2}}} \Phi(\hat{\boldsymbol{a}}(\epsilon), \epsilon) \hat{a}_{l_{2}, j_{2}}^{\prime}(\epsilon)+\frac{\partial^{2}}{\partial a_{l_{1}, j_{1}} \partial \epsilon} \Phi(\hat{a}(\epsilon), \epsilon)=0 \\
\sum_{l_{2}, j_{2}} \frac{\partial^{2}}{\partial a_{l_{1}, j_{1}} \partial a_{l_{2}, j_{2}}} \Phi(\hat{\boldsymbol{a}}(\epsilon), \epsilon) \hat{a}_{l_{2}, j_{2}}^{\prime \prime}(\epsilon)+\sum_{l_{2}, j_{2}} \sum_{l_{3}, j_{3}} \frac{\partial^{3}}{\partial a_{l_{1}, j_{1}} \partial a_{l_{2}, j_{2}} \partial a_{l_{3}, j_{3}}} \Phi(\hat{\boldsymbol{a}}(\epsilon), \epsilon) \hat{a}_{l_{2}, j_{2}}^{\prime}(\epsilon) \hat{a}_{l_{3}, j_{3}}^{\prime}(\epsilon)+ \\
2 \sum_{l_{2}, j_{2}} \frac{\partial^{3}}{\partial a_{l_{1}, j_{1}} \partial a_{l_{2}, j_{2}} \partial \epsilon} \Phi(\hat{\boldsymbol{a}}(\epsilon), \epsilon) \hat{a}_{l_{2}, j_{2}}^{\prime}(\epsilon)=0
\end{gathered}
$$

Differentiating this expression repeatedly and evaluating in $\epsilon=0$ gives a series of linear equation systems for $a^{\prime}(0), a^{\prime \prime}(0), \ldots$

As an example problem for illustrating the method, we let the full objective function be

$$
\Phi^{1}(\boldsymbol{a})=\sum_{k, i} \bar{y}_{k, i}-y_{k, i}-y_{k, i} \log \left(\bar{y}_{k, i} / y_{k, i}\right)+R(\boldsymbol{a}) .
$$

Here, $y_{k, i}$ is the measured counts in energy bin $k$ for projection ray $i, R(\boldsymbol{a})$ is a regularizer term and

$$
\bar{y}_{k, i}=\sum_{i^{\prime}} \int_{0}^{E_{\max }} S_{i^{\prime}}(E) h_{k, i^{\prime}, i}(E) \mathrm{e}^{-\sum_{l} A_{i^{\prime}, l} f_{l}(E)} \mathrm{d} E
$$

is the expected counts in energy bin $k$ for ray $i$, where $S_{i^{\prime}}(E)$ is the number of incident photons per energy incident on detector pixel $i^{\prime}$ and $h_{k, i^{\prime}, i}(E)$ is a bin- and energy-dependent point-spread function. Here, we 
have introduced the notation $A_{i, l}=\sum_{j} P_{i j} a_{j, l}$ where $P=\left(P_{i j}\right)$ is the system matrix of the forward projection operation, giving the projection of pixel $j$ along ray $i$. We then let the simplified objective function be

$$
\Phi^{0}(\boldsymbol{a})=\frac{\beta_{D}}{2} \sum_{i}\left((P \boldsymbol{a})_{i}-\hat{\boldsymbol{A}}_{i}^{R}\right)^{T} \Sigma_{i}^{-1}\left((P \boldsymbol{a})_{i}-\hat{\boldsymbol{A}}_{i}^{R}\right)+R(\boldsymbol{a})
$$

where $\beta_{D}$ is a tuning parameter. $\hat{\boldsymbol{A}}^{R}$ (a vector of $L I$ elements where $I$ is the number of projection rays) is an initial estimate, which we take to be the result of a ray-wise maximum-likelihood material decomposition ${ }^{4}$ without pixel-to-pixel cross talk in the forward model. Furthermore, $\Sigma_{i}$ is an estimate of the covariance matrix between the decomposed basis projections in ray $i$, given by the Cramér-Rao lower bound. ${ }^{5}$

Differentiating $\Phi(\boldsymbol{a}, \epsilon)$ gives

$$
\begin{gathered}
\frac{\partial^{2}}{\partial a_{l_{1}, j_{1}} \partial a_{l_{2}, j_{2}}} \Phi(\boldsymbol{a}, \epsilon=0)=\beta_{D} \sum_{i} P_{i, j_{1}} P_{i, j_{2}}\left(\Sigma_{i}^{-1}\right)_{l_{1}, l_{2}}+\frac{\partial^{2}}{\partial a_{l_{1}, j_{1}} \partial a_{l_{2}, j_{2}}} R(\boldsymbol{a}) \\
\frac{\partial^{2}}{\partial a_{l_{1}, j_{1}} \partial \epsilon} \Phi(\boldsymbol{a}, \epsilon)=\sum_{k} \sum_{i} \sum_{i_{1}} P_{i_{1}, j_{1}} \frac{\partial \bar{y}_{k, i}}{\partial A_{i_{1}, l_{1}}}\left(1-y_{k, i} / \bar{y}_{k, i}\right) \\
-\beta_{D} \sum_{l^{\prime}} \sum_{i} P_{i j_{1}}\left(\Sigma_{i}^{-1}\right)_{l_{1}, l^{\prime}}\left(\sum_{j} P_{i j} a_{j, l}-\hat{A}_{i, l^{\prime}}^{R}\right)+\frac{\partial(R(\boldsymbol{a})-R(\boldsymbol{a}))}{\partial a_{l_{1}, j_{1}}} \\
\frac{\partial^{3}}{\partial a_{l_{1}, j_{1}} \partial a_{l_{2}, j_{2}} \partial \epsilon} \Phi(\boldsymbol{a}, \epsilon)=\sum_{i} \sum_{i_{1}} \sum_{i_{2}} P_{i_{1}, j_{1}} P_{i_{2}, j_{2}} \sum_{k}\left(\frac{\partial^{2} \bar{y}_{k, i}}{\partial A_{i_{1}, l_{1}} \partial A_{i_{2}, l_{2}}}\left(1-y_{k, i} / \bar{y}_{k, i}\right)-\frac{\partial \bar{y}_{k, i}}{\partial A_{i_{1}, l_{1}}} \frac{\partial \bar{y}_{k, i}}{\partial A_{i_{2}, l_{2}}} \frac{y_{k}}{\bar{y}_{k, i}^{2}}\right) \\
+\beta_{D} \sum_{i} P_{i, j_{1}} P_{i, j_{2}}\left(\Sigma_{i}^{-1}\right)_{l_{1}, l_{2}}+\frac{\partial^{2}(R(\boldsymbol{a})-R(\boldsymbol{a}))}{\partial a_{l_{1}, j_{1}} \partial a_{l_{2}, j_{2}}}
\end{gathered}
$$

and similar expressions for higher-order derivatives.

For the regularizer term, we use an edge-preserving penalty penalizing nearest neighbors.

$$
R(\boldsymbol{a})=\sum_{l} \sum_{j} \sum_{j^{\prime}} g_{j, j^{\prime}, l} \phi\left(a_{j, l}-a_{j^{\prime}, l}\right)
$$

with

$$
g_{j, j^{\prime}, l}=\lambda \cdot\left\{\begin{array}{l}
1, j^{\prime} \in \mathcal{N}(j) \\
0 \text { otherwise }
\end{array}\right.
$$

where $\lambda$ is the regularization strength and $\mathcal{N}(i)$ is the set of abutting neighbors to pixel $i$ and

$$
\phi(x)=\frac{x^{2}}{2} .
$$

To illustrate the method, we used the CatSim ${ }^{6}$ package to simulate photon-counting CT acquisition of a FORBILD head phantom, with the fine structures in the inner ear removed. All tissues were assumed to have the same attenuation coefficient as water up to a density scale factor. We simulated the acquisition with $120 \mathrm{kVp}$ and $100 \mathrm{~mA}, 1000-\mathrm{mm}$ source-to-detector distance, and 500-mm source-to-isocenter distance, using a pre-patient filter modeled after the medium bowtie filter of the GE Lightspeed VCT* scanner. 1200 detector cells and 984 projection angles were used. For these initial results, we used a simplified forward model with a single basis

\footnotetext{
*Trademark of General Electric Company
} 
(a) FBP of raywise decomp.

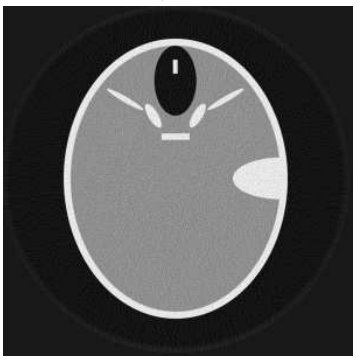

(d) FBP of raywise decomp. error

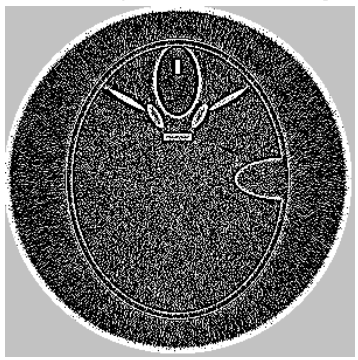

(b) $\Phi_{0}$ solution

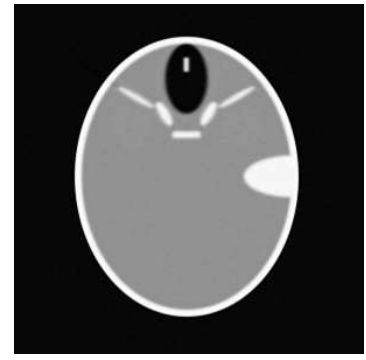

(e) $\Phi_{0}$ solution error

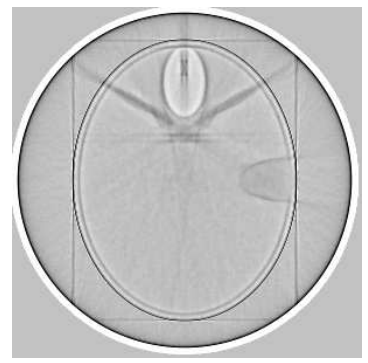

(c) Order 2 series exp

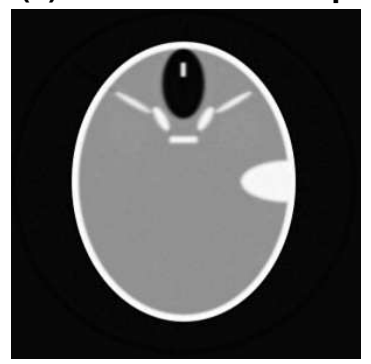

(f) Order 2 series exp error

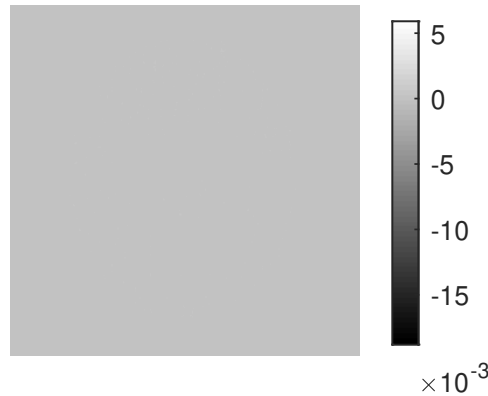

Figure 1. Reconstructed images: (a) FBP image. (b) Initial image (solution of $\Phi^{0}$ ). (c) Image generated from second-order series expansion. (d-f) Errors of images (a-c) relative to the converged reference image.

function (water) and a single energy threshold (20 keV), using a spectral response model for a 3-mm thick CdTe sensor with $(0.5 \times 0.5)-\mathrm{mm}^{2}$ pixels and $3 \mathrm{-keV}$ RMS energy resolution. ${ }^{7}$ The initial estimate $\hat{\boldsymbol{A}}^{R}$ was obtained using ray-wise maximum-likelihood basis estimation. The parameter $\beta_{D}$ was set to 1 and the regularization strength $\lambda$ was set to 1600 . The images were reconstructed on a $300 \times 300$ grid of $(1 \times 1)-\mathrm{mm}^{2}$ pixels.

In our model, the point-spread function is shift-invariant i.e. a function of $i^{\prime}-i$. For each view angle, the expected counts were convolved with this point-spread function. Poisson noise was then simulated based on the expected counts.

For each order in the Taylor expansion, an equation system of the form $H_{\Phi^{0}} \boldsymbol{a}^{(n)}=\boldsymbol{b}$ needs be solved, where $H_{\Phi^{0}}$ is the Hessian of $\Phi^{0}$ (See Eqs.2-3). This was solved using the preconditioned conjugate-gradient method implemented as pcg in Matlab R2018b (The Mathworks, Inc., Natick, MA, USA). To speed up convergence, we used a preconditioner operator of the form $M=D\left[\kappa_{j}\right] C\left[\kappa_{j}\right]$, selected to approximate $H_{\Phi^{0}}$, where $D\left[\kappa_{j}\right]$ is a diagonal matrix with elements $\kappa_{j}=\sqrt{\frac{\sum_{i} P_{i j} W_{i}}{\sum_{i} P_{i j}}}$. C is a convolution (implemented as a Fourier filtering) with the filter kernel $H_{\Phi^{0}} \boldsymbol{e}_{j}$ where $\boldsymbol{e}_{j}$ is a unit vector corresponding to a single pixel in the center of the field of view. This can be seen as a simplified, approximate version of the preconditioners described in Refs. 8 and 9 . All pixels outside the circular field of view were forced to be zero.

We first generated an initial reconstructed image $\hat{\mathbf{a}}(0)$ of equivalent water density with the quadratic $\Phi^{0}$. We then generated a second-order series expansion for the reconstructed water density image $\hat{\mathbf{a}}(1)$. The results were compared to a converged solution of the full objective function $\Phi^{1}$, calculated with 2000 iterations of an iterative reconstruction method $(\mathrm{BFGS})^{10}$ implemented in the ODL software package. ${ }^{11}$

\section{RESULTS}

Results are shown in Figs. 1 and 2. Fig. 1 shows the images obtained from filtered back-projection, from the simplified objective function $\Phi^{0}$ and from the second-order series expansion. This figure also shows the difference to the ground truth, obtained from optimizing $\Phi^{0}$ iteratively until convergence. 
(a)
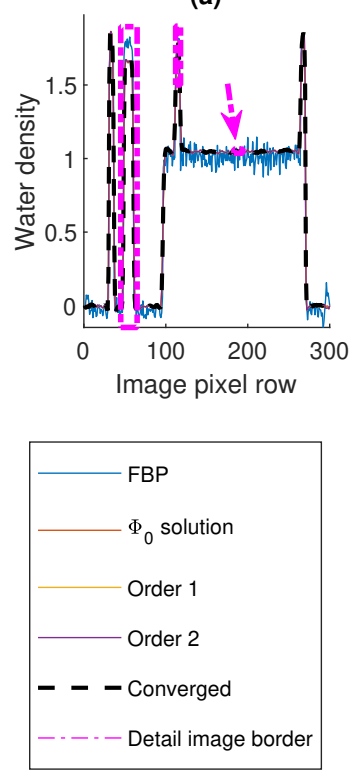

(b)

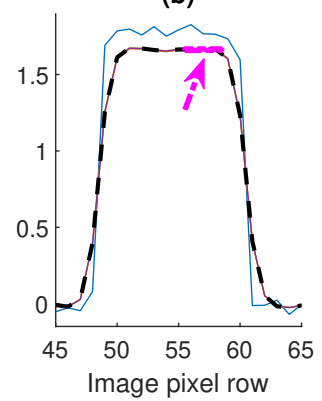

(e)

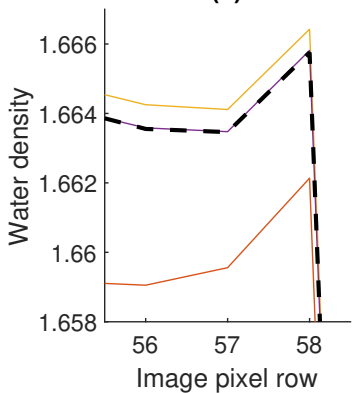

(c)

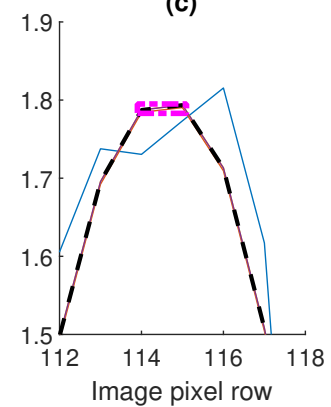

(f)

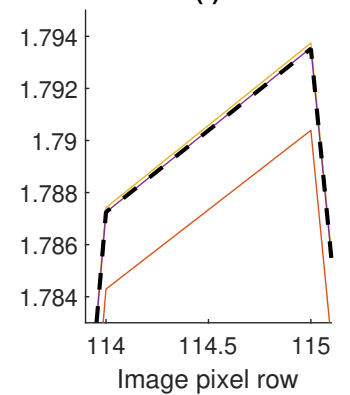

(d)

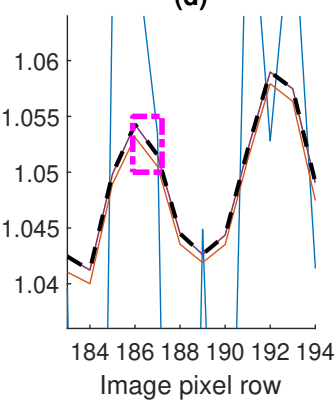

(g)

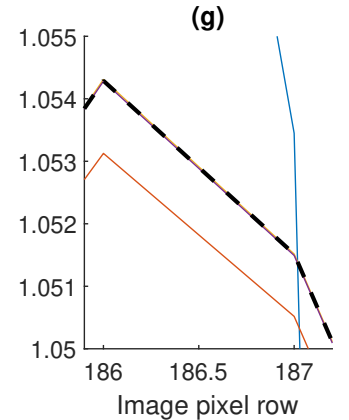

Figure 2. Vertical profiles through the center of the reconstructed images in Fig. 1, showing the initial estimate and images resulting from a first- or second-order expansion, as well as the converged reference solution ("converged"). (a) Full profile. (b-d) Detail profiles corresponding to the boxes in (a). (e-g) Zoomed-in views of the detail profiles corresponding to the boxes in (b-d), respectively.

Fig. 2 shows vertical profiles through reconstructed images with filtered backprojection, solution of $\Phi^{0}$, and first-and second-order series expansions, together with the converged solution. Also shown in the figure are zoom-in plots of bone structures and of a homogeneous part of the brain. Note that the plotted quantity is the relative density of water, meaning that a difference of $10^{-3}$ corresponds to $1 \mathrm{HU}$.

\section{DISCUSSION}

The magnitude of the second-order correction is in general smaller than the magnitude of the first-order correction, as can be seen from Fig. 2 where the first-order correction reduces the error to below $1 \cdot 10^{-3}$ (corresponding to $1 \mathrm{HU})$. With two correction terms, the maximum error anywhere in the image is $7.3 \cdot 10^{-4}$ compared to $1.7 \cdot 10^{-3}$ in the solution obtained from $\Phi^{0}$ (Fig. 1).

Our results show that the proposed series expansion method has the potential to reduce complicated, nonquadratic reconstruction problems to a series of quadratic optimizations. With optimized algorithms for solving quadratic reconstruction problems, reaching sufficient accuracy in 5-10 iterations per correction term instead of the present 80-100 iterations may be feasible. ${ }^{12,13}$ Further improvement of the new algorithm and comparison with existing algorithms will be needed in order to establish for which applications the proposed algorithm is best suited.

\section{ACKNOWLEDGMENTS}

This project has received funding from the European Union's Horizon 2020 research and innovation programme under the Marie Sklodowska-Curie grant agreement No 795747. Mats U. Persson discloses financial interests: Stockholder and consultant, Prismatic Sensors AB; Mats U. Persson is currently a visiting researcher at GE Research, Niskayuna, NY 12309, USA, with funding from the EU Research Executive Agency. 


\section{REFERENCES}

[1] Willemink, M. J., Persson, M., Pourmorteza, A., Pelc, N. J., and Fleischmann, D., "Photon-counting ct: Technical principles and clinical prospects," Radiology 289(2), 293-312 (2018). PMID: 30179101.

[2] Taguchi, K. and Iwanczyk, J. S., "Vision 20/20: Single photon counting x-ray detectors in medical imaging," Medical Physics 40(10), 100901 (2013).

[3] Thibault, J.-B., Sauer, K. D., and Bouman, C. A., "Newton-style optimization for emission tomographic estimation," Journal of Electronic Imaging 9(3), 269 - 282 (2000).

[4] Roessl, E. and Proksa, R., "K-edge imaging in x-ray computed tomography using multi-bin photon counting detectors," Physics in Medicine and Biology 52, 4679-4696 (jul 2007).

[5] Roessl, E. and Herrmann, C., "Cramér-rao lower bound of basis image noise in multiple-energy x-ray imaging," Physics in Medicine and Biology 54, 1307-1318 (feb 2009).

[6] De Man, B., Basu, S., Chandra, N., Dunham, B., Edic, P., Iatrou, M., McOlash, S., Sainath, P., Shaughnessy, C., Tower, B., and Williams, E., "CatSim: a new computer assisted tomography simulation environment," Proceedings of SPIE 6510, 856 - 863 (2007).

[7] Persson, M. and Pelc, N. J., "Simulation model for evaluating energy-resolving photon-counting ct detectors based on generalized linear-systems framework," Proceedings of SPIE 10948, 109481V (2019).

[8] Fu, L., Man, B. D., Zeng, K., Benson, T. M., Yu, Z., Cao, G., and Thibault, J.-B., "A preliminary investigation of 3D preconditioned conjugate gradient reconstruction for cone-beam CT," Proceediongs of SPIE 8313, 1051 - 1059 (2012).

[9] Fessler, J. A. and Booth, S. D., "Conjugate-gradient preconditioning methods for shift-variant pet image reconstruction," IEEE Transactions on Image Processing 8, 688-699 (May 1999).

[10] Griva, I., Nash, S. G., and Sofer, A., [Linear and Nonlinear Optimization], Siam (2009).

[11] Adler, J., Kohr, H., and Öktem, O., "Operator discretization library (odl)." https://github.com/odlgroup/odl.

[12] Fu, L., Yu, Z., Thibault, J.-B., Man, B. D., McGaffin, M. G., and Fessler, J. A., "Space-variant channelized preconditioner design for 3d iterative ct reconstruction," Proceedings of The 12th International meeting on Fully 3D Image Reconstruction in Radiology and Nuclear Medicine (2013).

[13] Kim, D., Ramani, S., and Fessler, J. A., "Combining ordered subsets and momentum for accelerated x-ray ct image reconstruction," IEEE Transactions on Medical Imaging 34, 167-178 (Jan 2015). 